\title{
COLORES Y LUCES DE LOS ESTADOS UNIDOS DE AMÉRICA. UNA VISIÓN DEL EXITOSO PINTOR VIAJERO JOSÉ ARPA A TRAVÉS DE LA PRENSA
}

\author{
COLORS AND LIGHTS OF THE USA. A VISION OF \\ THE SUCCESSFUL TRAVELLING PAINTER JOSÉ ARPA \\ THROUGH PRESS
}

\author{
Carmen Rodríguez Serrano \\ rodriguezserranocarmen@gmail.com
}

\begin{abstract}
El presente artículo tiene la intención de dar a conocer uno de los aspectos menos conocidos del pintor sevillano José Arpa, su etapa en los Estados Unidos de América. A través de la valoración desprendida de la prensa sevillana y texana, así como del análisis de algunas de sus obras de este periodo, se profundizará en una de las personalidades artísticas más interesantes del panorama artístico hispalense durante la primera mitad del siglo XX.

Palabras claves: Pintura, Estados Unidos de América, Texas, Prensa, José Arpa.
\end{abstract}

This study intends to show one of the less known aspects from Sevillian painter José Arpa, which is his stage in the United States of America. One of the most interesting artistic personalities in Seville during $20^{\text {th }}$ century can be deepened through the positive appraisals in the Sevillian and Texan press as well as the study of his paintings in his period in Texas.

Keywords: Painting, United States of America, Texas, Press, José Arpa.

Dice el dicho que nadie es profeta en su propia tierra, y sin duda, son numerosos los ejemplos y lugares donde esto sucede. En el mundo de la cultura y concretamente en el del arte, es frecuente encontrar casos en los que el artista es reconocido tras su muerte y otros casos en los que su obra es puesta en valor en vida, pero lejos de su propia tierra, de su lugar de origen. Esta última circunstancia, es la que ha motivado la redacción de este artículo, centrado específicamente en la valoración y reconocimiento del pintor sevillano José Arpa Perea durante la primera mitad del siglo XX en los Estados Unidos de América, donde era 
conocido como "The Sunshine Man", gracias a su habilidad para captar los reflejos del sol. Por ello, era necesario el estudio de su figura a través de las obras que realizó fuera de su propia tierra y sobre todo a través del análisis de algunos ejemplos de la prensa española y estadounidense, fuentes que encierran una importantísima información acerca de su personalidad artística y del éxito alcanzado al otro lado del Atlántico. La prensa sevillana, desde muy pronto, supo admitir el talento de la pintura de Arpa y hubo críticos que se lamentaron de la escasa valoración que desde su tierra se le había profesado, hecho que provocó su marcha a América. Por otro lado, la prensa norteamericana, y sobre todo la tejana, sí abordó desde un primer momento, la excelente acogida que tuvo el artista en el país y el éxito cosechado en la ciudad de San Antonio. Es frecuente incluso, encontrar fragmentos de prensa en los que Arpa es reconocido como un pintor tejano, uno de los representantes del Early Texas Art.

Como ya se ha señalado, este trabajo ha pretendido destacar y subrayar las opciones que países como Estados Unidos a finales del siglo XIX y principios del $\mathrm{XX}$, ofrecieron a numerosos artistas que tuvieron que emigrar de sus países en busca del reconocimiento y futuro de su obra. El país de las oportunidades también lo era en el mundo del arte, alejándose de las estrictas normas y estilos que imperaban en lugares de gran tradición artística, regidos por las Academias Nacionales de Bellas Artes. Arpa nos sirve para comprender y poner en valor las relaciones que se establecieron durante la primera mitad del siglo XX entre los artistas europeos y los propios americanos, enriqueciendo de manera extraordinaria el panorama cultural estadounidense.

El estudio, enfocado desde un punto de vista intercultural, marcado por el análisis de una personalidad artística con rasgos hispánicos y americanos, a través de su producción en Estados Unidos; así como desde un punto de vista interdisciplinar, debido a la investigación a través de fuentes visuales, bibliográficas, hemerográficas y documentales. Han sido numerosas las obras recopiladas y aún más los artículos y recortes de prensa consultados para entender las circunstancias artísticas del pintor, por lo que era necesario realizar una selección idónea que ilustrara los objetivos de este ensayo, así como introducir los aspectos biográficos del artista.

José Arpa Perea nació en Carmona, un pequeño pueblo situado en la provincia de Sevilla en 1858. De origen humilde, se cree que su formación inicial tuvo lugar junto a un pintor local de temática religiosa, de escasa trascendencia, pasando pronto a la capital, Sevilla, según Cascales ${ }^{2}$, a la edad de diez años, donde además lo señalará como pintor de brocha gorda, trabajo que le servía para subsistir mientras tomaba clases nocturnas en el Museo. El joven Arpa pudo por fin,

${ }^{1}$ FORRESTER-O'BRIEN, Esse: Art and Artists of Texas, Dallas, 1935.

2 CASCALES Y MUÑOZ, José: Sevilla Intelectual. Sus Escritores y Artistas Contemporáneos, Madrid, 1896. 
terminar asistiendo a la Escuela de Bellas Artes, donde tuvo la oportunidad de formarse junto a pintores de la talla de Eduardo Cano ${ }^{3}$ o Manuel Wssel de Guimbarda, dentro de la estética del Romanticismo.

Como era frecuente entre los jóvenes artistas españoles, Arpa participó en certámenes oficiales y exposiciones ${ }^{4}$, y fue becado por la Diputación de Sevilla en el año 1883, para realizar una estancia de aprendizaje en Italia ${ }^{5}$. En este país y concretamente en la ciudad de Roma, tuvo la ocasión de codearse con otros grandes artistas que marcaron notablemente su pintura, así como influenciarse de la fuerte impronta orientalista que había dejado el magnífico pintor Mariano Fortuny. A su vuelta a Sevilla en 1886, monta su propio taller donde inició, además, su actividad docente, aunque sin llegar a obtener del todo el reconocimiento esperado. En ese momento, José Arpa tenía un estilo claramente definido, con una tendencia hacia el Realismo, caracterizado por una espléndida riqueza cromática, así como por el gusto hacia la pintura de paisaje ${ }^{6}$. Será en esos años, además, cuando se relacione con la Escuela de paisajistas de Alcalá ${ }^{7}$, que practicaban la pintura al Plein Air, convirtiéndose a partir de ese momento el paisaje, en el género predilecto del artista ${ }^{8}$.

${ }^{3}$ Emmanuel Benezit escribió en su Dictionaire critique et documentaire des peintres, Sculpteurs, Dessanateurs et Graveurs..., que Eduardo Cano de la Peña falleció en 1807, circunstancia que advierte Cecilia Steinfeldt y que cita en A Spanish Artist in San Antonio, una lectura que ofreció a la Texas State Historical Association Meeting en San Antonio. La investigadora señala que si esa era la fecha de defunción de Cano, no fue posible el encuentro entre el maestro y el discípulo. No obstante, hay que desmentir tal afirmación, ya que Eduardo Cano falleció en Sevilla en el año 1897, siendo por lo tanto y sin duda, uno de los profesores de José Arpa.

${ }^{4}$ Cualquier joven artista que se preciase, tenía que participar en los certámenes oficiales que organizaban las academias de Bellas Artes, instituciones que regían e imponían las normas y cánones artísticos. Ello supuso en muchos casos el estancamiento del arte, ya que no había opciones para introducir novedades y aires renovadores dentro de este mundo.

${ }^{5}$ En ese momento la capitalidad artística en Europa pasa progresivamente a París, aunque Italia y concretamente Roma, seguían acogiendo a un gran número de pintores, escultores y arquitectos de todas partes del mundo.

${ }^{6}$ Este género, además del retrato y la pintura de historia, serán los grandes protagonistas del XIX en España, así como lo fue el Realismo desde la segunda mitad de ese siglo, estilo que perdurará hasta bien entrado el XX en Andalucía y que rompía, no de forma definitiva, con el Romanticismo.

${ }^{7}$ Escuela de pintores que se reunió hacia 1890 en torno a la figura de Emilio Sánchez Perrier, para pintar al Plein Air los alrededores del Río Guadaira y de la Vega de Carmona, en la provincia de Sevilla. Fueron conocidos como el Barbizon español y entre sus integrantes se encuentran Manuel García Rodríguez, José Lafita y José Rico Cejudo, entre otros.

${ }^{8}$ Es necesario indicar como Arpa va a pintar paisajes, en un primer momento, influenciado por la Escuela de Alcalá, pero tras su estancia en México y Estados Unidos, su pintura se diferenciará notablemente del resto de representantes de esa agrupación. 
Ya se ha indicado como el pintor, a pesar de recibir encargos e iniciar su labor docente ${ }^{9}$, no va a obtener todo el éxito esperado, llegando incluso a realizar otras actividades artísticas, como la que le llevó a participar como corresponsal gráfico en la Guerra de Margallo o Primera Guerra del Rif, que tuvo lugar en Marruecos. Tras pasar una serie de años en Sevilla, con la esperanza de conseguir nuevas oportunidades y movido por la búsqueda de otros paisajes, el pintor abandonará su país poniendo rumbo a México en torno a $1897^{10}$. Decía Cicerón que la observación de la naturaleza y la meditación generan el arte, y sin duda, ello debe ser aplicado a la obra de un gran número de artistas. Esa afirmación, llegó a convertirse en el motor y en el estilo de vida de muchos pintores y en concreto del que ocupa estas líneas, el cual vivió en una constante persecución de la luz, del color, de la naturaleza, que junto a su carácter inquieto, le convertirán en un artista viajero.

En México, gracias a su propio talento y a la ayuda de la familia Quijano, que actuó como su mecenas principal, permanecerá hasta 1910, cuando con motivo de la Revolución Mexicana marche a los Estados Unidos. En ciudades como Jalapa y Puebla, va a desarrollar una interesantísima producción, donde los estudios atmosféricos, de color y de luz predominen en sus composiciones. El paisaje será el género cultivado principalmente, aunque no el único, y con el que participe en exposiciones tan destacadas como la XXIII Exposición Nacional de la Escuela de Bellas Artes de México, en el año $1898^{11}$, o la Exposición Nacional de Bellas Artes organizada

${ }^{9}$ Impartía clases de dibujo a algunos jóvenes que iniciaban su andadura artística, entre ellos Javier Winthuysen.

${ }^{10}$ En torno a la fecha de partida, se han barajado diferentes posibilidades, aunque la más probable parece ser la del año 1897. Juan Fernández Lacomba, autor del catálogo realizado en la primera gran exposición monográfica realizada sobre José Arpa en Sevilla en el año 1998, sitúa al artista en México a partir de 1895. Fernando González Moreno, en su artículo "A Miguel de Cervantes: Don Quijote por José Arpa Perea", publicado en Cuadernos de Arte de la Universidad de Granada, la sitúa en 1896, al igual que Montserrat Galí, primera persona que abordó la figura de José Arpa en el contexto mexicano con su artículo "José Arpa Perea en México (1895-1910)", del año 2000. Según se ha podido investigar, el pintor firma en Sevilla en el año 1897 dos ilustraciones, concretamente las del tomo VII de Tradiciones Sevillanas de Manuel Cano y Cueto, obra en la que ya había intervenido en años anteriores. A ello, se suma el hecho de que Cascales hable de Arpa en Sevilla, en tiempo presente, en 1896, dentro de su obra Sevilla Intelectual, por lo que se supone imposible que este autor desconociera que Arpa se encontraba en México desde 1895.

${ }^{11}$ En esta línea hay que señalar como la idea tan difundida entre la bibliografía norteamericana de que Arpa marchó a México para ocupar un cargo en la Escuela Nacional de Bellas Artes, es bastante improbable, ya que no aparece como profesor en ésta, no hay documentación que lo pruebe y además, su obra estuvo mucho más vinculada a la ciudad de Puebla. Si hubiera sido profesor, no habría aparecido tan modesta y discretamente en las críticas de la XXIII Exposición de la Escuela Nacional de Bellas Artes de 1898. Muy esclarecedor, a este respecto, resulta el fragmento obra de Angélica Velázquez Guadarrama, 
por el Círculo Católico de Puebla en 1900, donde Arpa será además junto al pintor mexicano Daniel Dávila, uno de los organizadores ${ }^{12}$. En la primera de ellas, su participación fue bastante modesta para la crítica, y no será hasta su establecimiento en la ciudad de Puebla, cuando alcance buenos comentarios. A pesar de obtener el tan ansiado éxito en esta ciudad, José Arpa necesitó algunos años más para lograr la total valoración de su obra en un contexto menos provinciano. Dicho reconocimiento, llegaría a partir del año 1910, cuando con motivo de la Revolución Mexicana marchó a los Estados Unidos ${ }^{13}$, donde se estableció durante más de veinte años, aunque sin dejar de realizar viajes frecuentes a España. Hay que señalar como desde muy pronto, los propios investigadores norteamericanos le incluyeron dentro de la nómina de artistas texanos, sin dejar de reconocer su origen español. Autores como Frances Battaille Fisk ${ }^{14}$, Caroline Remy ${ }^{15}$ o Forrester O'Brien ${ }^{16}$, motivados por su incansablemente dedicación al paisaje texano y americano, por las fuertes influencias que recibió de pintores de la talla de los Onderdonk, así como por su pertenencia a grupos de la intelectualidad texana, le aceptaron como un pintor autóctono más.

El primer contacto entre Arpa y los Estados Unidos tuvo lugar en el año 1893, cuando el Gobierno Español mandó cuatro obras del pintor a la Columbian

en su tesis de licenciatura La Presencia del Arte Español en la XXIII Exposición de la Escuela Nacional de Bellas Artes, 1898-1899 y que dice lo siguiente: “(...)De los contingentes de artistas llegados a México en el siglo pasado, el español, fue, por razones obvias, el más importante y numeroso, pues sin contar a los catalanes Pelegrí Clavé y Manuel Vilar y posteriormente a Antonio Fabrés - contratados ex profeso por la Academia de San Carlos para reestructurar la enseñanza-, por su cuenta y riesgo llegaron Carlos Paris, Lorenzo Cereza, Manuel Serrano, Lorenzo de la Hidalga, Manuel Ilizaliturri. Francisco Mas, Esteban Landaluze, José Cusachs, José Escudero y Espronceda y en los albores del siglo XX, Pierre de Rivera, José Arpa, Félix Vía y Manuel Beltrán de Quintana (...)”. De él se puede extraer que Arpa no tuvo ningún papel como profesor en la capital mexicana.

12 OLIVERA BANCHERO, Cecilia Beatriz: La Exposición de Bellas Artes organizada por el Círculo Católico de Puebla en 1900, México, D.F., 2011. (Tesis inédita)

${ }^{13}$ Son varios los autores que señalan que José Arpa fue a San Antonio en numerosas ocasiones antes de 1910, en un primer momento, como profesor y supervisor de los hijos de Antonio Quijano, su mecenas en Puebla. Arpa se había encargado de la educación artística de ellos en México, así que no es de extrañar que fuera así. Sí sabemos que en 1910 acompañó a la familia Quijano a Estados Unidos, cuando con motivo de la Revolución mexicana, abandonaron el país, estableciéndose finalmente en New Rochelle, Nueva York, momento en el que Arpa siguió fiel a su instinto viajero, y continuó viajando hasta establecerse definitivamente en San Antonio.

${ }^{14}$ BATTAILE FISK, F. A: History of Texas Artists and Sculptors, Austin, 1986.

15 REMY, Caroline: Biographical Sketches of Fifteenth Texas Artists, San Antonio, Texas, 1966.

${ }^{16}$ FORRESTER-O'BRIEN, Esse: op. cit. 
Exposition in Chicago, recibiendo estas una buena acogida ${ }^{17}$. Por otro lado, son numerosas las fuentes que indican como en el año 1899, el artista pasará por la ciudad de San Antonio, donde expuso en la Feria de Arte de 1900, aunque es seguro que regresó a México, sin ser hasta 1923, cuando fije su residencia allí. En un primer momento, Arpa viajará por todo el país, captando las impresiones, la naturaleza y los reflejos lumínicos de estados tan diferentes como Nueva York, Wisconsin o Arizona, aunque será Texas, el lugar donde se establece definitivamente. El grandioso paisaje norteamericano impactó al pintor y así quiso capturarlo, en todo su esplendor. Como se ve a través de su obra, Arpa pinta según le dicta su alma y según se muestra la realidad. Sin duda, la esencia del realismo hispánico está presente en su obra, aunque es un error etiquetar su obra. Imbuido de numerosas influencias y conocedor de diferentes estilos y pinceles, nuestro pintor capta la naturaleza y la luz según él la percibe. De la observación de sus paisajes se puede indicar como se aleja de la pintura realizada por otros paisajistas sevillanos como Emilio Sánchez Perrier o Manuel García Rodríguez, mostrándose mucho más próximo, en sus años de madurez, a la pintura cultivada por artistas como Julian Onderdonk, aunque sin abandonar nunca esa esencia propia, quizás adquirida a través de la experiencia fruto de sus viajes por tan dispares lugares. En esta línea, resultan muy interesantes las palabras que Cecilia Steinfeldt ${ }^{18}$ dedicó a su obra y con las que define la esencia de su arte:

"Arpa's painting style was some what more flamboyant tan the Onderdonks. A brilliant colorist, he was enamoraded with the fervent sunlight and atmospheric luminosity of the radiant Texas country side. He found interest and beauty in earthy, ordinary scenes that most artists would have disdained. He could turn a simple barnyard, rock quarry group of Irish Flat huts into sparkling works of art"19.

La investigadora subrayó esa capacidad del pintor al captar la esencia luminosa propia de Texas, así como su facilidad para hacer bello lo aparentemente ordinario. Steinfeldt había señalado, además, que los más destacados artistas que trabajaron en dicho estado en la segunda mitad del siglo XIX eran de origen europeo, circunstancia que remarca la posterior influencia del pintor sevillano en San Antonio, así como la buena acogida a los talentos externos que enriquecieron al Early Texas Art $^{20}$. Son varios los pintores que se consideran parte de

${ }^{17}$ Estas obras pertenecen a la etapa en la que José Arpa define su estilo tras su regreso de Roma. Alguna de ellas sigue anclada a la tradición de la pintura de historia, mientras que otras muestran el estilo del Arpa que más tarde pintará en Estados Unidos.

${ }^{18}$ STEINFELDT, Cecilia: "Lone Star Landscapes: Early Texas through Artist's Eyes”, M3, San Antonio Museum Association, 1986.

${ }^{19}$ Ibídem.

${ }^{20}$ Se trata del arte producido por los artistas que habían nacido, vivido o trabajado en Texas desde la segunda mitad del siglo XIX hasta 1950 aproximadamente (Principalmente 
la transición hacia este movimiento. Uno de los más importantes es Robert Onderdonk, el cual llegó a Texas procedente de Nueva York, para convertirse en la piedra angular del arte texano, aunque será su hijo, Julian Onderdonk el que realmente alcance una alta cotización. Ambos, sin duda, pueden ser considerados como los más influyentes pintores de la historia texana, aunque no los únicos. De la mano de estos, José Arpa fue considerado otro de sus prominentes artistas, convirtiéndose además, en maestro de muchos de los pintores representantes del ya citado Early Texas Art. Su carácter sociable le hizo ganar numerosos amigos entre los intelectuales y los artistas locales, como el fotógrafo Ernst Raba o los ya mencionados Onderdonk, junto a los que formó grupos culturales de encuentros. En el San Antonio Express, con fecha de 27 de diciembre de 1936 y bajo el titular "San Antonio Scenery Supplies Inspiration for Artists" se presentó una interesante fotografía (Figura 1) que recoge el estrecho vínculo que adquirió Arpa con los artistas e intelectuales más destacados de San Antonio. La nota de prensa presenta lo siguiente:

"A recent visit to the Studio of Ernest Raba brought forth a very interesting photograph of a group of artists who were gathered as guests of the late Julian Onderdonk at his home in East French Place en 1914. In this group, as shown in the picture, are left to right, standing Mr. Amick, Julian Onderdonk, Ernest Raba, Theodore Fletcher, Tom Brown, Joe Braun, Robert Leo Cotton; seated Ernest A. Thomas, Robert J. Onderdonk, José Arpa, Charles Simmang, Alois Braun (... $)^{21}$ ".

En efecto, ésta reunión de intelectuales muestra al grupo conocido como The Brass Mug Club, en un encuentro que tuvo lugar en 1914 en la casa de Julian Onderdonk. Entre ellos había pintores, un fotógrafo, un profesor de violín, otro de música y un grabador. Pero este no fue el único grupo al que perteneció Arpa, ya que tal y como se observa en una fotografía de 1913, también acostumbró a reunirse con artistas de la talla de Robert Leo Cotton, Rolla Taylor y Erns Raba. The Gang, tal y como era conocido este grupo, tenía entre sus integrantes a miembros del primer club mencionado.

Sintiéndose como pez en el agua, José Arpa fijó su residencia en la ciudad de San Antonio en el año 1923. Plenamente inmerso en el ambiente cultural de la ciudad, abrió su estudio y escuela de arte, a la que se unió su sobrino, el joven pintor Xavier González, con la intención de ayudar a su tío. Tras varios cambios de ubicación, la escuela se fijó definitivamente, en la vieja casa Vance, situada en New Street, en 1927. Entre sus alumnos, figuraron pintores como John Griffith,

los tempranos años de comienzos del siglo XX). Ellos van a conformar un estilo propio que simboliza las señas de identidad del propio arte texano, donde el estudio de la naturaleza va a imperar sobre otros géneros.

${ }^{21}$ SAN ANTONIO EXPRESS, "San Antonio Scenery Supplies inspiration for Artists", 27/12/1936. 
Porfirio Salinas o el escultor Octavio Medellín, aunque se puede decir que fueron dos los pintores que realmente absorbieron los rasgos impresionistas de la pintura de Arpa, Rolla Taylor y Carl T. Hoppe ${ }^{22}$.

El contexto cultural que presentaba la ciudad elegida por Arpa para establecerse, era bastante favorable para el arte, pero se dio además una circunstancia, que fue la que realmente marcó la diferencia. No se puede entender el panorama artístico en Texas y San Antonio sin mencionar las Texas Wildflowers Competitive Exhibitions, que fueron según Cecilia Steinfeldt, un hito en la historia del arte texano. Creado por el millonario dedicado a los negocios del petróleo Edgar B. Davis, este concurso que se inició en 1927 y tuvo su última edición en 1929, dio los premios económicos más altos que en ningún concurso se habían dado. Ello muestra el alto valor que alcanzó el arte y la pintura en este estado durante el siglo XX. En estas competiciones se reunieron numerosos artistas como Dawson Dawson-Watson, Peter Hohnstedt o José Arpa, que fue galardonado en las tres ediciones llevadas a cabo.

Tras años de idas y venidas a España, en $1932^{23}$ José Arpa regresa a su tierra, con la idea de pasar allí sus últimos días, sin saber que el destino le depararía veinte años más de existencia. Durante esos años, el pintor fue objeto de numerosos homenajes y elogiosos artículos, donde se destacaba principalmente su condición viajera, siendo Carmona, su pueblo, donde siempre fue muy querido y apreciado, uno de los que más le ofreció. Al fallecer en 1952, dejó a sus espaldas un imponente y disperso corpus artístico que trasluce su esencia pictórica.

\section{JOSÉ ARPA A TRAVÉS DE SU OBRA EN LOS ESTADOS UNIDOS DE AMÉRICA}

Tras el análisis de sus circunstancias biográficas y de su condición viajera, el estudio de algunas de sus obras va a convertirse en uno de los instrumentos más destacados para probar su relación con los Estados Unidos. Con este fin, han sido seleccionadas una serie de pinturas ejecutadas entre 1910 y 1929, así como alguna obra que bien siendo anterior en el tiempo, muestra los rasgos pictóricos propios en la obra de José Arpa. A Mexican Wash Day-Tierra Caliente (Figura 2), obra realizada hacia 1900, revela el estilo maduro que Arpa había desarrollado en México, imbuido, no obstante, por las corrientes lumínicas y paisajistas texanas, las cuales ya había tenido oportunidad de conocer. Esta pintura, que se encuentra en Estados Unidos, presenta una técnica habitual en algunas de las obras del pintor, incluso en algún ejemplo de los años finales de su vida. Es una

${ }^{22}$ Éste estudió con Arpa la pintura al Plein Air, cerca de Bandera a finales de los años 20.

${ }^{23}$ Según Cecilia Steinfeldt esta es la fecha en la que abandonó San Antonio aunque Juan Fernández Lacomba la sitúa en 1935. 
magnífica composición donde se recoge un motivo recurrente en Arpa, y que representa a mujeres lavando la ropa en un entorno fluvial. El estudio de la perspectiva, así como del color y la luz, nos hablan de un artista excepcional, que pinta sin recetas, sin sometimiento a algún estilo y que sigue los dictados de sus ojos y de los viajes que se ponen ante estos. En esta línea, tal y como se señaló, durante los primeros años de estancia en los Estados Unidos, José Arpa va a pintar numerosos paisajes de diferentes estados, reflejando las acusadas diferencias atmosféricas que entre estos existen. En el estado de Wisconsin y concretamente en Prairie du Chien, va a realizar una acuarela sobre el Río Mississippi ${ }^{24}$ (Figura 3 ), que firma en 1912 y que muestra el interés del pintor por los paisajes fluviales en todas sus variedades ${ }^{25}$. El estudio cromático utilizado en el agua, así como el del espacio natural, revelan los reflejos del oscurecido cielo, típico de un día gris, y que dista mucho de los realizados en México y posteriormente en Texas. De ese mismo año, es el lienzo Árbol en Wisconsin (Figura 4), que nuevamente capta ese tipo de atmósfera oscurecida, fría, destacando en un primer plano la figura de un árbol, castigado por el invierno que revela una de las características principales en su pintura, la de los encuadres forzados y las composiciones aparentemente aleatorias.

On the River Bank (Figura 5), donde se repite esa característica, es un lienzo fechado hacia 1916-1923, que ejemplifica nuevamente el interés de Arpa por captar el paisaje fluvial. En primer término y jugando con la composición incluye la figura de un niño campesino, el cual mira directamente al espectador, estableciéndose una fuerte relación visual. Tanto el estudio lumínico, como el colorido, muestran a un artista influenciado por la pintura impresionista tejana, que es capaz de mezclar con su propia tradición. La obra, que diversas fuentes indican que pudo ser realizada en uno de sus viajes a Sevilla ${ }^{26}$, trasluce la fuerza telúrica que supo imprimir Arpa a las rocas y piedras representadas, elementos cargados de gran realismo.

Con mucha más seguridad, en uno de sus viajes a Sevilla en torno a 1920, Arpa pintó Lavanderas en Villanueva del Río (Figura 6). Ésta representa una escena en la que nuevamente aparecen una serie de mujeres lavando en un río, en un pequeño pueblo de la provincia de Sevilla. La escena, recuerda tanto en la temática como en la pincelada, a $A$ mexican Wash Day. La pincelada vibrante, cargada de materia, es la que define la escena y las figuras se componen a través de manchas color. En esta línea hay que subrayar que no solo los paisajes

\footnotetext{
${ }^{24}$ Ésta está dedicada a Carmela Quijano.

${ }_{25}$ José Arpa pintará de manera incansable a lo largo de toda su vida, el río Guadalquivir.

${ }^{26}$ A pesar de que así es aceptado por diversas fuentes, parece ser que ésta, es una obra tejana en toda regla. El paisaje, la vestimenta del campesino, etc, son muy dispares a lo que pudo ver Arpa en Andalucía.
} 
fluviales despertaron el interés del pintor, sino que también lo hicieron los marítimos, que del mismo modo ofrecían semejantes posibilidades lumínicas. Fechada hacia 1924 y firmada en la ciudad texana de Galveston, Galveston Dry Dock (Figura 7) es una interesantísima obra que muestra, en este caso, un paisaje marítimo. Arpa despliega en ella un gran abanico cromático para representar el reflejo de los barcos y cabañas del muelle en el agua. El humo que sale de las chimeneas de los barcos y la atmósfera gris, recuerdan a la pintura de Turner y a la de los impresionistas franceses. Es frecuente encontrar temas recurrentes en la obra de Arpa, tal y como ya se ha indicado y así sucede con este lienzo, ya que se conserva una visión del Puerto de Nueva York, realizado por este mismo artista, con el que guarda muchos paralelismos.

En la línea, nuevamente de los impresionistas franceses, José Arpa va a realizar una serie de diferentes vistas del Gran Cañón de Arizona, captado a diferentes horas del día y desde diferentes perspectivas. Son varios los ejemplos existentes, encontrándose algunos de ellos en Sevilla. El Gran Cañón (Figura 8) firmado en los Estados Unidos propiedad del Museo de Bellas Artes de Sevilla y que se fecha en torno a 1925, es un excelente ejemplo paisajístico donde se puede apreciar el ya citado espléndido manejo que del color y la luz tenía Arpa. Esta pintura, proporciona además, al espectador la misma fuerza telúrica que caracteriza al Cañón del Colorado. Al analizar la obra se observa el minucioso estudio lumínico realizado por el pintor y como el atardecer distorsiona las formas y colores del paraje. De este mismo momento es Piedras movibles (Figura 9), obra firmada de nuevo en los Estados Unidos de América y que capta el Gran Cañón del Colorado desde una perspectiva diferente. En este caso, la formación geográfica es interrumpida visualmente por unas grandes piedras móviles, situadas en un primer plano. Del mismo modo que vimos en la obra anterior, los colores y el estudio lumínico es excepcional, aunque en este caso son algo más agresivos, más intensos. Lo cierto, es que Arpa los representó tal y como los vio. Los atardeceres allí parecen irreales, de ensueño y así fueron captados.

Entre las obras más conocidas de Arpa en Texas, se encuentran los campos de flores, motivo que más tarde promovió Edgar B. Davis en sus Texas Wildflower Competitive Exhibition, uno de los motores más importantes para el arte texano en esos años. En la primera edición de la Texas Wildflower Competitive Exhibition, celebrada en 1927, el pintor va a participar con Verbena, obra que resultó premiada y que es un magnífico ejemplo de la perfección que adquirió el arte de Arpa en San Antonio. Este campo de verbenas florecido, invita a la observación, a la profundización. Sin duda, es uno de los mejores estudios de color de toda la obra del sevillano y uno de los más reproducidos junto con Chumberas en flor (Figura 10). Esta es una obra norteamericana que ejemplifica la confusión que desde Sevilla se ha mantenido con Arpa. A pesar de la creencia de que la pintura representa una escena andaluza, es más que evidente que refleja un paisaje árido texano. Realizada hacia 1928, esta pintura está totalmente relacionada con 
Cactus flowers, obra que fue galardonada con el tercer premio, de la Texas Wildflower Competitive Exhibition, celebrada en 1928 y que se encuentra en el San Antonio Art League Museum. Este tipo de representaciones, absolutamente texanas, influenciaron notablemente en otros pintores del Early Texas Art, discípulos de Arpa, tales como Hoppe. En ambas obras, la pincelada vibrante y la riqueza cromática encumbran a Arpa como el pintor de la luz y la naturaleza.

Del análisis de éstas obras, se puede extraer y percibir el estilo definido de Arpa, enriquecido por el estudio de la luz y sus viajes, siendo Estados Unidos, el lugar donde alcance el culmen de su carrera, llegando a ser considerado un artista texano más.

\section{JOSÉ ARPA A TRAVÉS DE LA PRENSA: UNA PUESTA EN VALOR DE SU PERSONALIDAD ARTÍSTICA}

La prensa ha sido junto al análisis de su obra, una de las fuentes claves para la concepción de este artículo. Tras realizar un breve recorrido por sus circunstancias artísticas, barajar diferentes datos frutos de los estudios de diferentes investigadores y analizar algunas de sus obras más destacadas en los Estados Unidos, hay que concluir con la opinión que despertó el pintor entre la prensa sevillana y texana. Algunos ejemplos bastan para advertir que José Arpa llegó a ser un artista plenamente integrado en los Estados Unidos, y que así se percibió desde muy pronto en su tierra, criticándose por el contrario, el escaso reconocimiento que en ella se le había profesado. A pesar de existir numerosos ejemplos, se ha seleccionado una breve muestra de los mismos:

En Arte y Artistas, el 28 de enero de 1905, José Rico Cejudo hizo la siguiente afirmación:

"En grave compromiso me encuentro al decidirme a hablar de este artista, pues ligándome a él lazos de amistad, pudieran desvirtuar el espíritu de mis crónicas, que es el de la más estricta justicia.

No trato de dar a conocer a un artista. Pepe Arpa, desde hace tiempo, brilla con luz propia, pues su principal cualidad, es ser personal en sus trabajos y este es el mayor mérito en el arte. (...) Luego, más tarde, pasó a México y Estados Unidos, donde Arpa supo sacar de la naturaleza grandiosa y exuberante de aquellos países, motivos para hermosas creaciones que le dieron honra y dinero, ganando también varias medallas de oro en diversas exposiciones, con merecida justicia que no supieron hacerle en su patria. (... $)^{27}$.

Imbuido por una gran carga crítica, Rico Cejudo va a honrar la figura del que además de buen pintor, era su amigo. Con una diferencia temporal acusada, aunque sin apartarse de la línea de este primer ejemplo, Luis Claudio Mariani, en el Diario Independiente el 3 de mayo de 1922, hará una excelente crítica acerca de la

${ }^{27}$ RICO CEJUDO, José: “José Arpa”, Arte y Artistas, Sevilla, 28/01/1905. 
personalidad artística del pintor en "La Exposición de Bellas Artes. Los Paisajes de José Arpa":

“(...) Apartado de reuniones - es decir, poco amigo de perder el tiempo-y sin solicitar nunca los honores de la letra de molde, don José Arpa realiza en silencio una labor de arte refinado y exquisito del que pocos paladares pueden apreciar su rareza. Arte en silencio, como dijo Mauclair, de finas percepciones, de agudezas sutiles, de extraña diversidad en su conjunto estilizado por un mismo nervio. Arte raro, porque obedece a una singular interpretación no ecléctica, sino fecundada por la misma naturaleza a la que parece amar este pintor, no en su aspecto artificial, sino en su realidad. (...) Obra independiente y original es la de don José de Arpa. Se ve alli la mano adiestrada a través de muchos años de práctica, responde a la traducción de la visión pictórica. Un delicado sentimiento, se diría que un estado de alma de dolor contenido, de serenidad cercana a la melancolía, palpita en las visiones de Arpa. (... $)^{28 "}$.

Sin duda, Mariani es uno de los más acertados escritores, entre los cuales abordaron el análisis del pintor. Crítico con otros artistas sevillanos de su generación, se observa, a través de sus palabras, la admiración que siente por Arpa, gracias al elogio que realiza sobre la original y personal obra del carmonense. Va a incidir sobre todo, en como el pintor nunca estuvo sujeto a etiquetas de estilos, sino que más bien pintó lo que le dictó su alma. Es un error etiquetar su pintura y aún menos encasillarla en una moda, ya que dentro de ésta, incluso, se encuentran ejemplos que parecen ser realizados por diferentes pinceles y que hablan de una personalidad artística heterogénea.

De sumo interés entre los artículos texanos, fue el publicado en el San Antonio Express el 23 de marzo de 1924, donde se expuso la alta cotización que había alcanzado la obra de José Arpa en los Estados Unidos, incluso llegando a generar disputas por la demanda de su trabajo en diferentes ciudades. El titular "Rival cities lure artista who loves San Antonio Sunshine" engloba dicha circunstancia:

"Galveston, Chicago and Los Angeles are angling for Jose Arpa, great Spanish colorist, to whom this city is home; What he would like to do to make this the art center of the State. Chicago and some of the cities in the West are angling for José Arpa (... $)^{29 "}$."

Es muy significativa la valoración extraída de la edición de mañana del San Antonio Express con fecha de 17 de octubre de 1926, donde se aborda la participación de Arpa en la decoración del Nuevo Teatro construido en la ciudad de San Antonio. El titular "Arpa, Noted Colorist, Depicts Texas of Old and of Today In

${ }^{28}$ MARIANI, Luis Claudio: "La Exposición de Bellas Artes. Los Paisajes de José Arpa”, La Unión, Sevilla, 03/05/1922.

${ }^{29}$ SAN ANTONIO EXPRESS, "Rival cities Lure Artist who loves San Antonio sunshine", 23/03/1924. 
San Antonio's New Theater" encierra halagos muy importantes a la obra del pintor, considerando a Arpa como el mejor colorista de América:

"When the new Texas theater opens late in November, it will have in its ornamentation eight mural paintings, four of these turning the pages back to pioneer days and the others depicting the Texas of today.

America's finest colorist, José Arpa, now is painting these panels, each of which measures 16 by 25 feet. In the entire South or Southwest there will be nothing like it for the paintings will reflect that radiant sunshine for which Arpa's work is noted. (... $)^{30 "}$.

Muy interesantes también resultan las palabras dedicadas por la prensa sevillana al artista, en sus años más prósperos en Texas. Así, Eduardo Paradas Agüera en El Correo de Andalucía, el 5 de noviembre de 1927, publicó "Artistas españoles en América”, artículo que incide nuevamente en el éxito alcanzado en Estados Unidos:

“(...) De allí pasó a los Estados Unidos y penetró en el Estado de Texas, recibiendo en todas partes sinceros testimonios de respeto y admiración. Pero principalmente, en la ciudad de San Antonio fueron tan numerosas y fervientes las solicitaciones de sus admiradores, que le indujeron a instalarse definitivamente alli, donde había vislumbrado ya el pintor un ambiente propicio para sembrar la semilla de su arte.

En breve tiempo, haciendo verdaderos alardes de su inteligencia y voluntad, logró fundar la única Academia de Pintura que existe en aquel Estado. A ella consagra actualmente todos afanes con un tesón y unas energías impropios de sus setenta y un años.

Los diarios de Texas dedican con frecuencia grandes espacios en sus editoriales a encomiar sus relevantes dotes personales, las excelencias de su pintura y su intensa propaganda artística y docente.

$Y$ no es precisamente el mero lucro lo que mueve y estimula al artista en sus arriesgadas empresas. Arpa es uno de esos hombres admirables para quienes la palabra "negocio" carece de sentido, quizás porque, como todo genio civilizador, vive instintivamente confiado en que ha de recibir, como don celestial e indefectible, su pan y su vino en todas partes. La obra civilizadora de Arpa en América no tiene otra mejor explicación.

Numerosísimos son ya los jóvenes matriculados en la Academia de Pintura de la W. Nueva 210 ST. que por el número de asistentes y por el trajín y entusiasmo que en ella reinan da la sensación de una extraordinaria vitalidad como fenómeno desconocido hasta ahora en aquellas latitudes $(. . .)^{31}$ ”.

Este artículo es de vital importancia ya que representa una valiosa fuente de información acerca del éxito que obtuvo la academia fundada por Arpa, así como de la valoración y respeto que en aquellas tierras se le profesó.

${ }^{30}$ SAN ANTONIO EXPRESS, "Arpa noted colorist, Depicts Texas of old and of today in San Antonio's New Theater", 17/10/1926.

31 PARADAS AGÜERA, Eduardo: "Artistas españoles en América", El Correo de Andalucía, Sevilla, 05/11/1927. 
En La Unión, el 6 de agosto de 1928, se publicó "El Laureado pintor Don José Arpa a su regreso de América", el periodista, del mismo modo que lo hizo Mariani y Paradas Agüera valora el personal estilo artístico de Arpa y resalta la justa cotización que existe de su obra en los Estados Unidos:

“(...) Resueltamente aniquilada la pintura vieja quedaba por reivindicar el prestigio de magníficos pintores que alcanzaron aquella época catastrófica, y, sin embargo, no se contagiaron de majadería.

Don José Arpa es uno de ellos. Fuerte, vibrante, colorista, de intensa espiritualidad carculante en el tiempo, sin pararse en sus años mozos, como hacen otros.

Ha sabido actualizarse, renovándose de continuo, prestando oído a los sermones de la estética de vanguardia, despreciando lo "pompier" y amando siempre un poco la ironía y cultivando un suave humorismo.

Hace unos cuantos años se fue a Norteamérica y allí tuvo su firma la cotización que merece.

Le rodearon discípulos, que recibían a diario sus sabias enseñanzas. Sus cuadros destacaron en las Exposiciones, y se habló de aquel español, doblemente por serlo sevillano, que se abría paso entre los nuevos a los setenta años.

Sugestiva, encantadora paleta la de este pintor que ha sido admirado en todo el mundo.

$Y$ ayer, en un convivio fraterno, nos decía don José Arpa que es necesario estimular las corrientes artísticas españolas hacia los Estados Unidos. Que es necesario que los artistas organicen exposiciones y lleven sus cuadros a los concursos y presenten sus obras en la sala tal y en el saló cual; pero que si todo eso es muy necesario y muy saludable al arte español, aún lo sería más, y desde luego con más eficiencia, que el Gobierno español organizara oficialmente una Exposición de pintura y escultura españolas con los Estados Unidos.

Nos lo decía don José Arpa recién regresado de la tierra de los dólares, de ese país dueño de la llave mágica para abrirse casi todas las puertas del mundo.

Sería oportuno que la ciudad hiciera algo en honor de este gran artista encanecido al servicio de una obra tan eminentemente patriótica y ciudadana como lo es el aportar un esfuerzo considerable en prestigio de la pintura española. ${ }^{32}$ "

A pesar de tratarse aspectos tan interesantes como la gran labor realizada por Arpa en San Antonio, así como el carácter renovador de su pintura, quizás uno de los artículos más interesantes, fue el publicado en El Liberal el 8 de agosto de 1928. "ARTISTAS SEVILLANOS QUE VUELVEN. Una conversación con José Arpa", recoge unas palabras de Arpa que alaban la labor de mecenazgo ejercida en Texas, consciente de lo diferente que resulta el patrocinio de arte en España:

${ }^{32}$ LA UNIÓN, "El laureado pintor José Arpa a su regreso de América", Sevilla, 06/08/1928.

LABORATORIO DE ARTE 28 (2016), pp. 543-564, ISSN 1130-5762

e-ISSN 2253-8305 - DOI http://dx.doi.org/10.12795/LA.2006.i.01.29 
"Desde hace unos días se encuentra en nuestra ciudad el excelente artista pintor don José Arpa y Perea, recién regresado de San Antonio de Tejas (Norte-América), donde tiene su residencia (...)

Arpa, que vive -que vive bien-en Norte-América prodigando su arte, viene ahora a Sevilla atraído por la proximidad del Certamen Ibero-americano, donde expondrá en unión de los más destacados artistas españoles. (...)

- ¿En qué ambiente se desenvuelve allá el arte? - le hemos preguntado.

- En muy favorable ambiente de protección; pero, dentro de él, en lucha con los elementos hebreos, que lo aprovechan mediando entre el productor y el comprador.

Un caso de protección - afirma Arpa:

Mr. Dawis, avecindado en Luling - pueblo próximo a San Antonio-destinó este año pasado cincuenta y dos mil dólares a premios entre los artistas de San Antonio, del Estado de Tejas y de Norte América; de los cuales premios obtuve el tercero, de mil quinientos dólares.

Es extraordinario el caso de este Mecenas. Arruinóse en investigaciones petroliferas, rehizo su fortuna y la agrandó hasta lo incalculable al hallar unos yacimientos en Luling, y hoy ha enriquecido a cuantos le ayudaron, en honor de los cuales dio recientemente una fiesta, congregándoles en el citado punto. Por cierto que también acudieron en tal día numerosos agentes vendedores de automóviles, que hicieron magnífico negocio con los nuevos ricos.

Este Mr. Dawis costea fastuosos banquetes anuales en honor de los artistas premiados, autoridades y personas más caracterizadas de San Antonio y sus proximidades, y tiene el buen gusto de obsequiar a las damas concurrentes con ramos de orquídeas que encarga a París.

- Un verdadero Mecenas.

- Indudablemente.

- ¿Tiene un alto precio por allá la pintura española?

- Todo lo español. España está de moda en Norte-América, y es lástima que no sepamos aprovechar la circunstancia $(\ldots)^{33}$ \%.

Sin duda, frases como la final, en la que José Arpa revela las amplias posibilidades que tienen los artistas españoles al otro lado del Atlántico, resumen en gran medida sus inquietudes artísticas. Él sí supo aprovechar dicha circunstancia y así ha quedado demostrado.

Este estudio de la obra del pintor elaborado a partir del análisis interdisciplinar de su pintura y de su valoración a través de la prensa escrita, ha llevado a determinar que a pesar de ser José Arpa un artista español, su estilo está absolutamente en la línea artística texana, además de ser Estados Unidos el país que desde muy temprano supo reconocer su talento. Este hecho, se mantiene hoy día debido a la fuerte demanda actual de la obra del pintor, en esta tierra, donde es altamente cotizado, a diferencia de España, donde perduran cuantiosos clichés

${ }^{33}$ EL LIBERAL, "Artistas sevillanos que vuelven, Una conversación con José Arpa", Sevilla, 08/08/1928. 
respecto a su figura y donde sigue siendo insuficientemente conocido. Sin duda, es necesario seguir profundizando en su obra, así como en el estudio de fuentes como la prensa, con el propósito de otorgar su merecido lugar a uno de nuestros pintores más internacionales.

Fecha de recepción: 30 de septiembre de 2015.

Fecha de aceptación: 20 de noviembre de 2015.

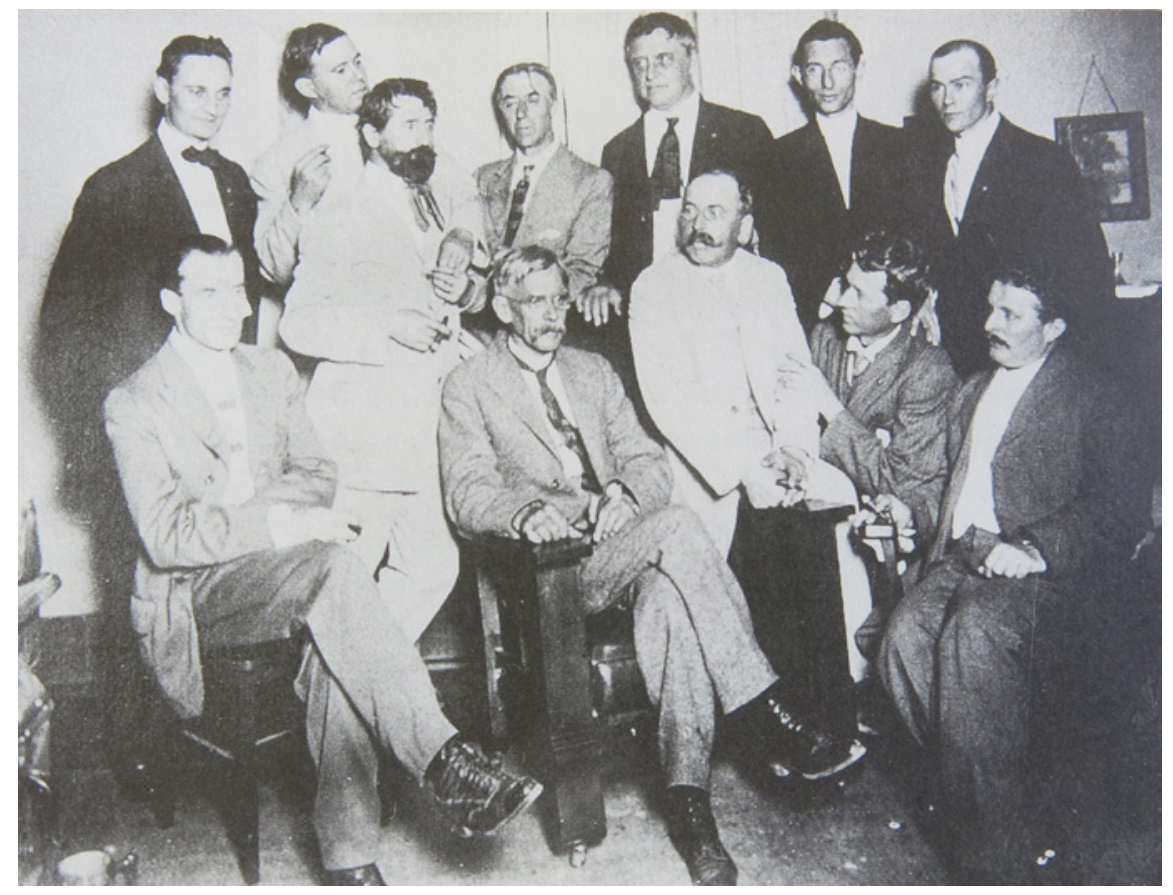

Figura 1. The Brass Mug Club, 1914, Photographs, SAMA Historical Photographic Archives, San Antonio, Texas. (Imagen extraída de STEINFELDT, Cecilia, Art for History's Sake. The Texas Collection of the Witte Museum, The Texas State Historical Association for The Witte Museum of San Antonio Museum Association, San Antonio, 1993). 


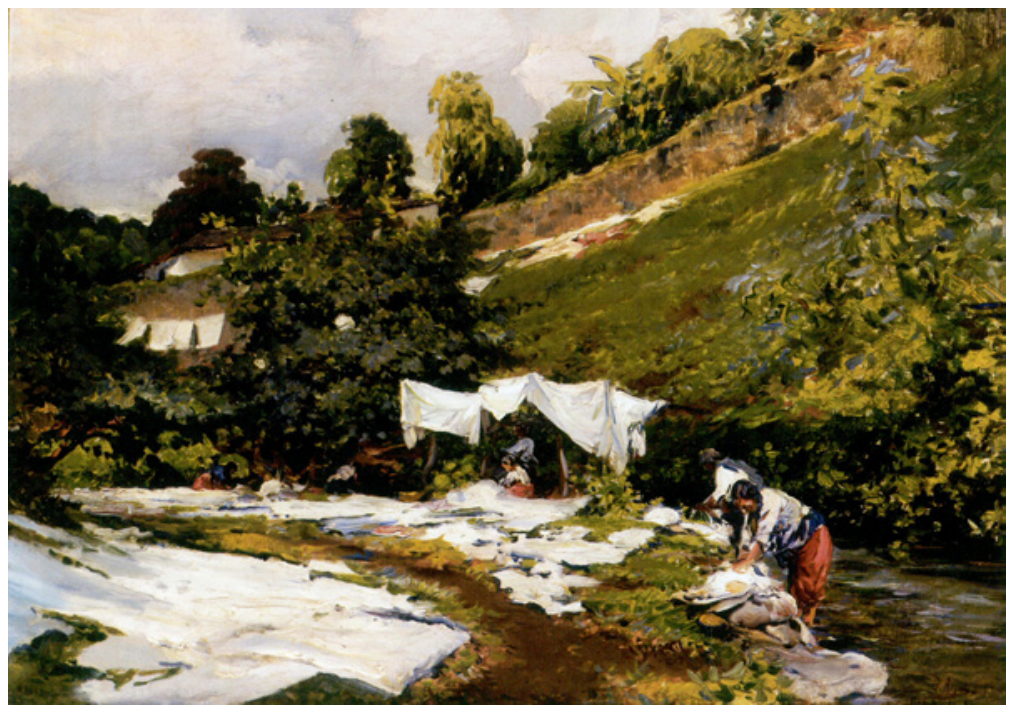

Figura 2. A Mexican Wash Day- Tierra Caliente, José Arpa Perea, hacia 1900. Óleo sobre lienzo, 35,5 x 50,8 cm, Collection of Beverly and George Palmer. (Imagen extraída del catálogo de la Exposición Texas Impressionism: Branding with Brushstroke and Color, 1885-1935, San Antonio, Texas, 2013).

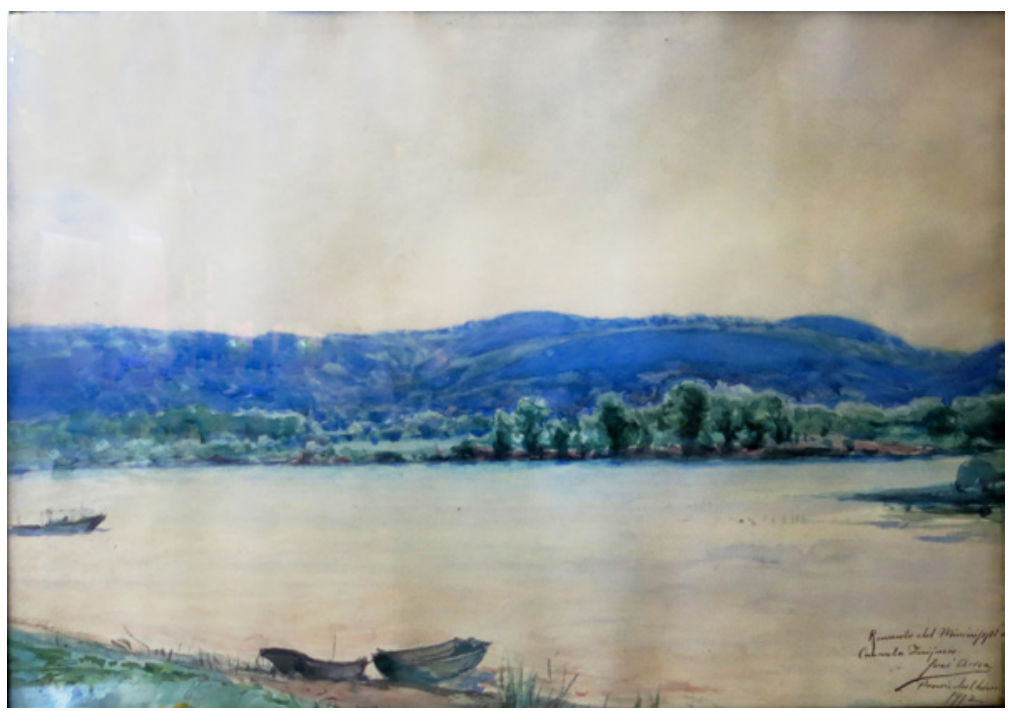

Figura 3. Vista del río Mississippi, José Arpa Perea, 1912. Acuarela sobre papel, 35,7 x 50,7cm, Colección Privada, Sevilla. 


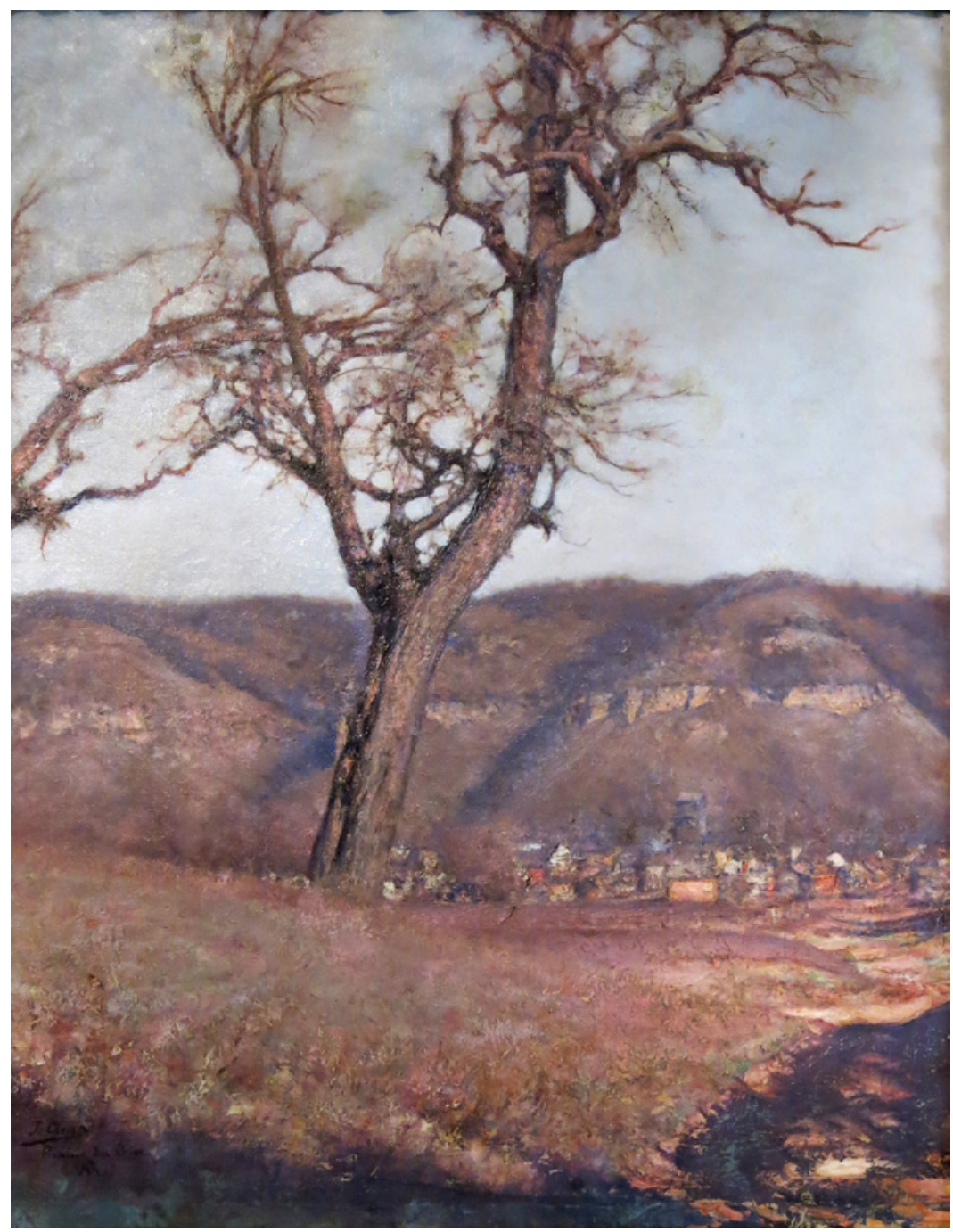

Figura 4. Árbol de Wisconsin, José Arpa Perea, 1912. Óleo sobre lienzo, 56,5 x 44 cm, Colección Privada, Estados Unidos. 


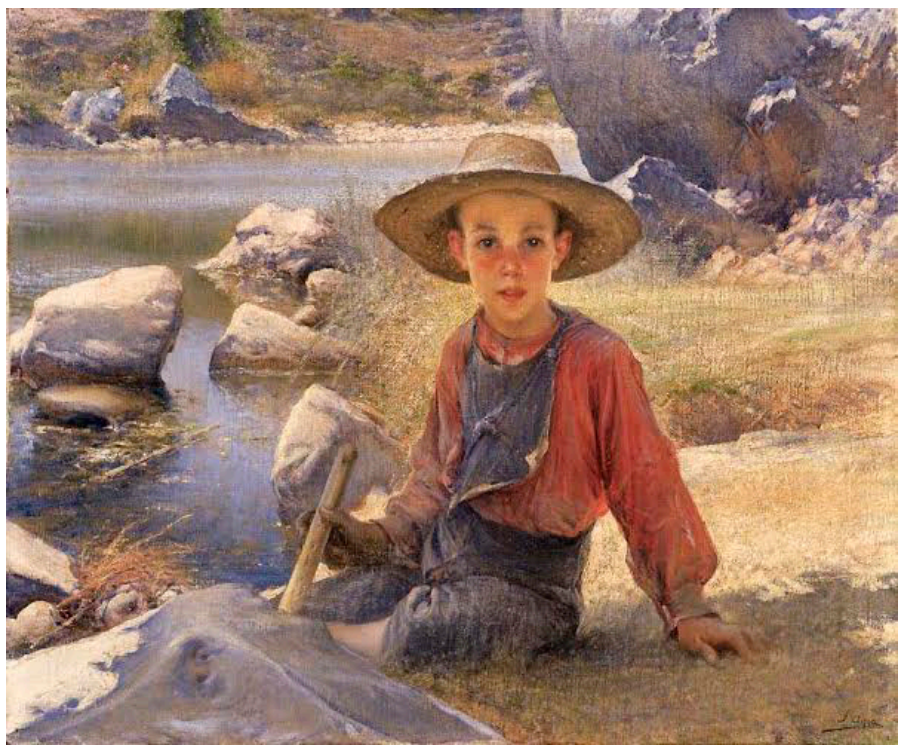

Figura 5. On the River Bank, José Arpa Perea, hacia 1929. Óleo sobre lienzo, 61x 73,7 cm, McNay Art Museum, San Antonio, Texas. (Imagen cedida por el museo).

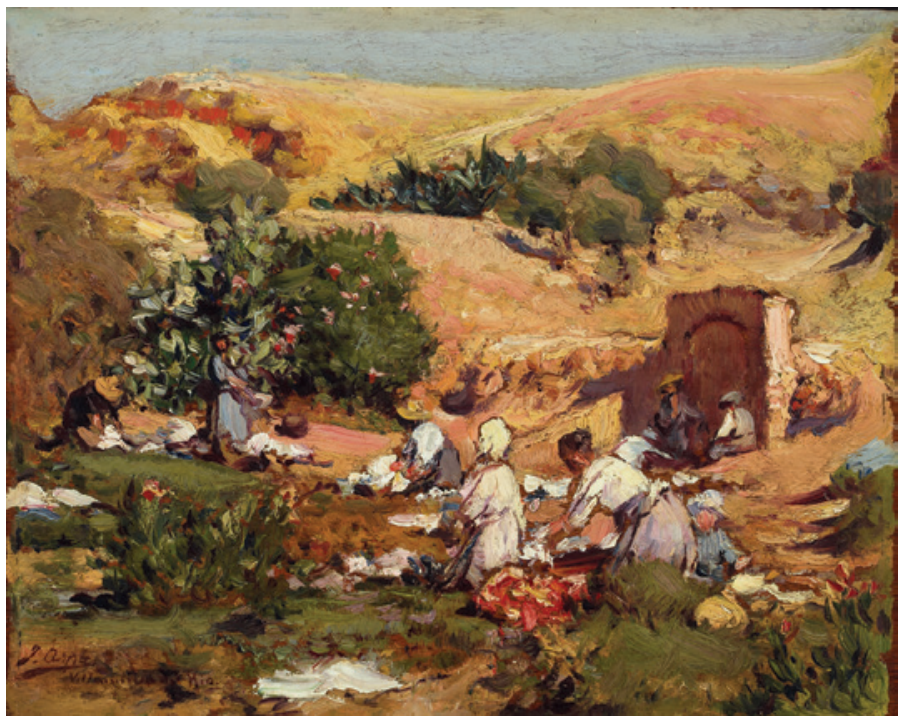

Figura 6. Lavanderas en Villanueva del Río, José Arpa Perea, hacia 1920. Óleo sobre tabla, 21,59 x 26,67 cm, Meadows Museum, Dallas, Texas. (Imagen cedida por el museo). 


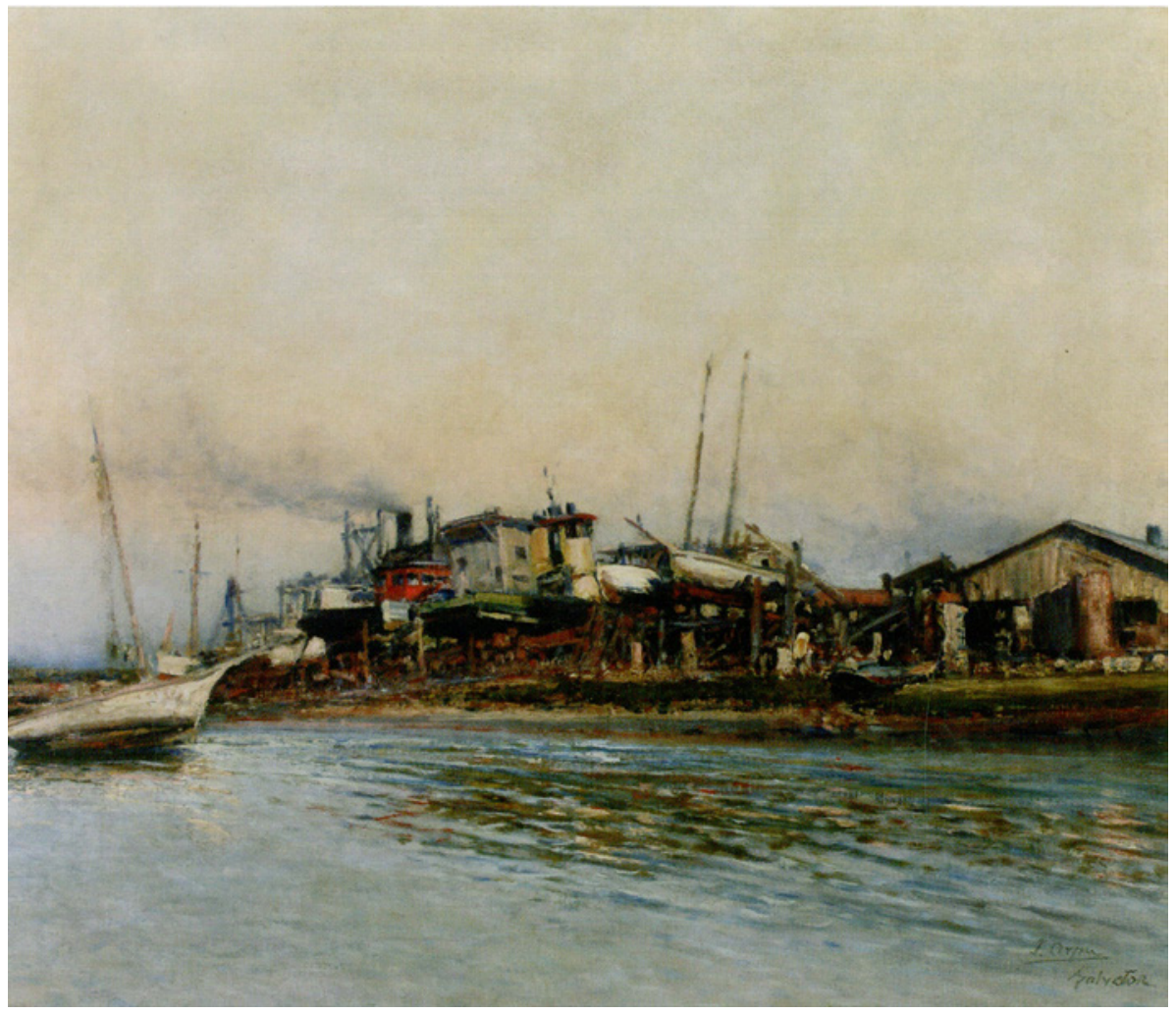

Figura 7. Galveston Dry Dock, José Arpa Perea, hacia 1925. Óleo sobre lienzo, 50,8 x 60,9 $\mathrm{cm}$, Collection of Linda and Williams Reaves. (Imagen extraída del catálogo de la Exposición Texas Impressionism: Branding with Brushstroke and Color, 1885-1935, San Antonio, Texas, 2013). 


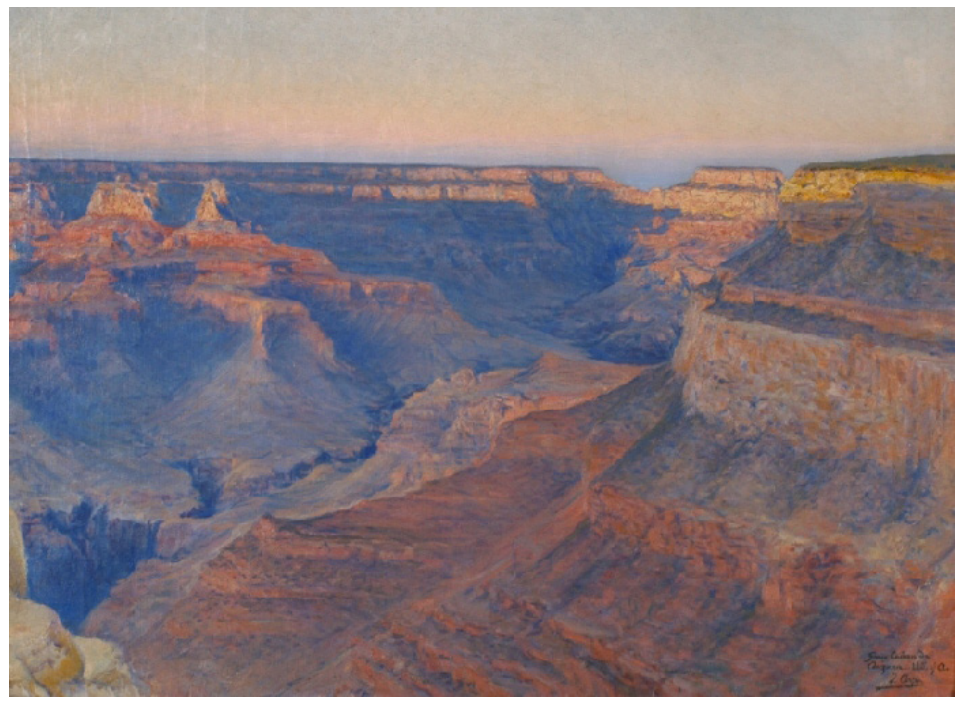

Figura 8. El Gran Cañón de Arizona iluminado a la puesta de sol, José Arpa Perea, hacia 1925. Óleo sobre lienzo, 76 x 102 cm, Museo de Bellas Artes, Sevilla.

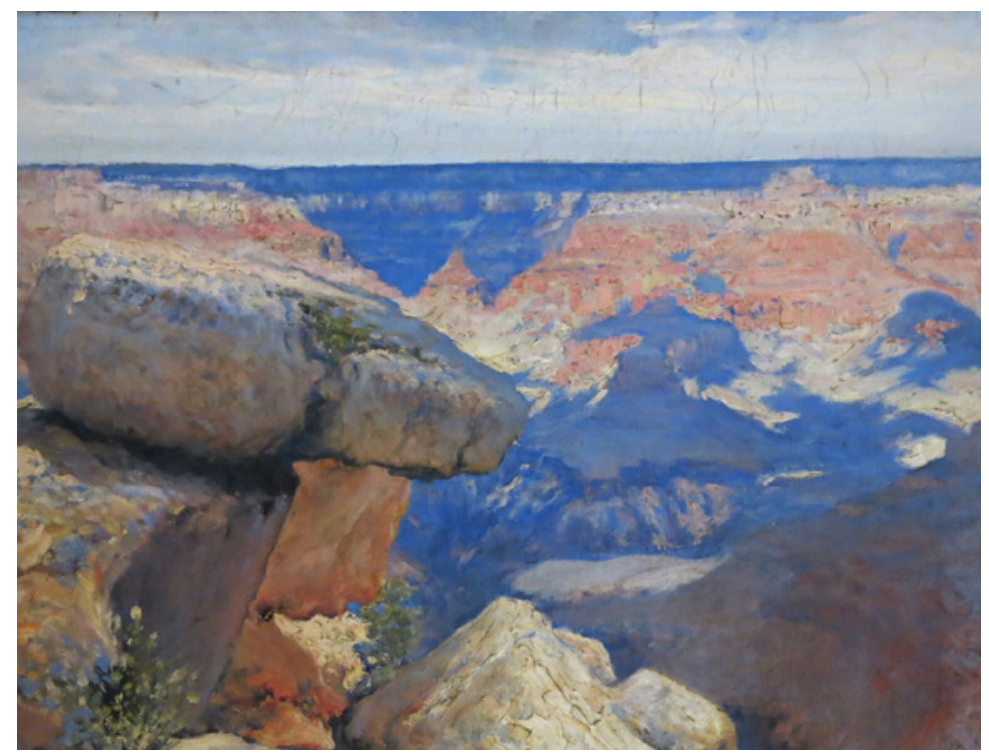

Figura 9. Piedras movibles del Gran Cañón del Colorado, José Arpa Perea, hacia 1925. Óleo sobre lienzo, 51 x $61 \mathrm{~cm}$, Real Academia de Bellas Artes de Santa Isabel de Hungría, Sevilla. 


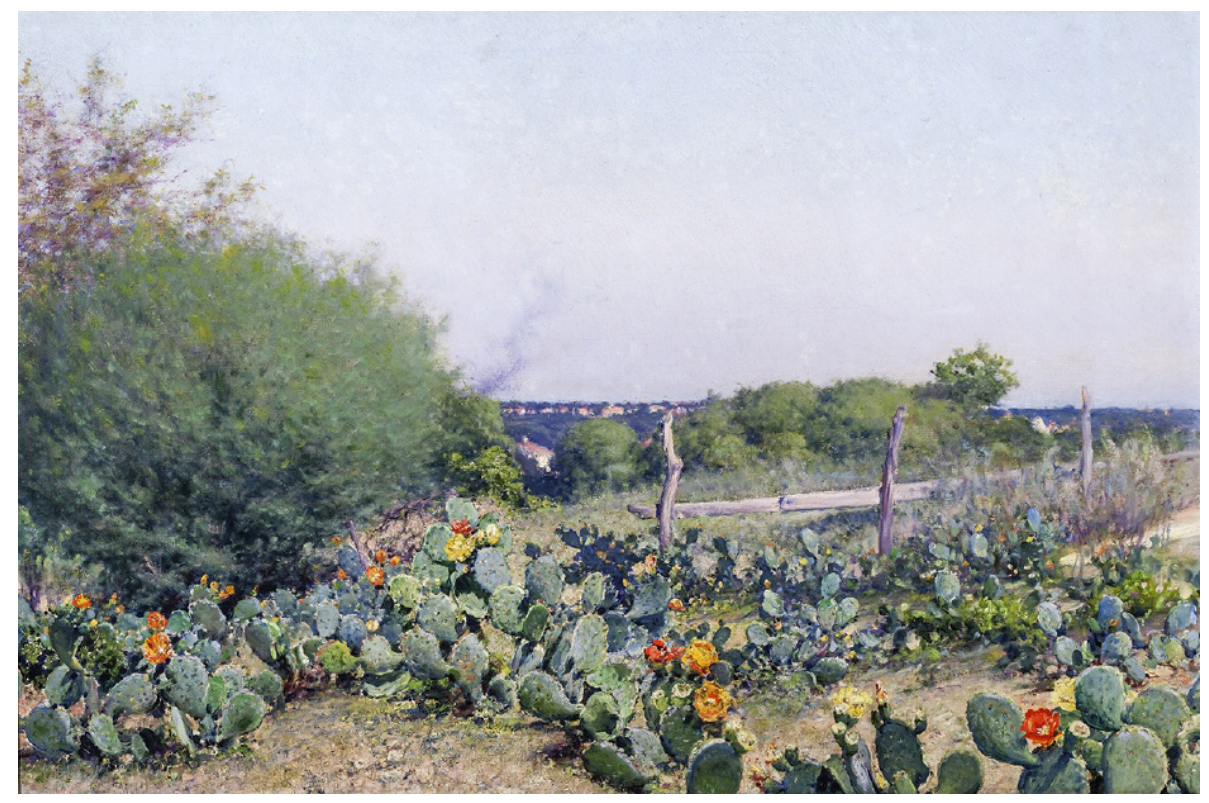

Figura 10. Cactus en flor, José Arpa Perea, 1928. Óleo sobre lienzo, 61,9 x 92 cm, Museo de Bellas Artes, Sevilla. 